\title{
Sensibilidade e especificidade da força de preensão manual como discriminador de risco para multimorbidades em idosos
}

\author{
Sensitivity and specificity of hand grip strength as a risk \\ discriminator for multimorbidities in the elderly
}

\author{
Loiamara Barreto Santos ${ }^{1}$, Alba Benemérita Alves Vilela, \\ Clarice Alves dos Santos², Rosângela Souza Lessa², Saulo Vasconcelos Rocha²
}

\begin{abstract}
Resumo
Objetivo: Selecionar, por meio da sensibilidade e da especificidade, os melhores pontos de corte da força de preensão manual como discriminador de risco para presença de multimorbidades em idosos. Método: Estudo transversal, realizado com 310 idosos com idade $\geq 60$ anos, de ambos os sexos, residentes no município de Ibicuí, Bahia. Para identificação da força de preensão manual como discriminador de risco para presença de multimorbidades, foi adotada a análise das curvas Receiver Operating Characteristic (ROC), com intervalo de confiança de 95\%. Posteriormente, foram identificados os pontos de corte com suas respectivas sensibilidades e especificidades. Resultados: A área total sob a curva ROC entre a força de preensão manual e a presença de multimorbidades foi de 0,59 (IC 95\% = 0,52-0,67) para mulheres e de 0,58 (IC 95\%=0,50-0,67) para homens. Os melhores pontos de corte para discriminar a presença de multimorbidade foram, para mulheres e homens, respectivamente, 15,35 kgf (sensibilidade de 69,7\% e especificidade de 52,2\%) e 24,8 kgf (sensibilidade de 49,5\% e especificidade de 68,4\%). Conclusão: Os resultados encontrados neste estudo sugerem que a força de preensão manual pode ser utilizada como discriminador de risco para presença de multimorbidades em idosos.
\end{abstract}

Palavras-chave: saúde do idoso; predição; morbidade.

\begin{abstract}
Objective: Select through sensitivity and specificity the best cutoffs for hand grip strength as a risk discriminator for presence of multimorbidities in the elderly. Method: A cross-sectional study was conducted with 310 elderly individuals aged $\geq 60$ years, of both genders, living in the municipality of Ibicuí, Bahia state, Brazil. Hand grip strength as a risk discriminator for presence of multimorbidities was identified by analysis of the Receiver Operating Characteristic (ROC) curves with $95 \%$ confidence interval. Subsequently, the cutoff points with their respective sensitivity and specificity values were identified. Results: The total area under the ROC curve between hand grip strength and presence of multimorbidities was $0.59,95 \% \mathrm{Cl}(0.52-0.67)$ for women and $0.58,95 \% \mathrm{Cl}(0.50-0.67)$ for men. The best cutoff points to discriminate presence of multimorbidities were $15.35 \mathrm{kgf}(69.7 \%$ sensitivity and $52.2 \%$ specificity $)$ and $24.8 \mathrm{kgf}$ (49.5\% sensitivity and $68.4 \%$ specificity) for women and men, respectively. Conclusion: Results suggest that hand grip strength may be used as a risk discriminator for presence of multimorbidities in the elderly.

Keywords: health of the elderly; forecasting; morbidity.

${ }^{1}$ Núcleo de Estudos em Saúde da População, Programa de Pós-graduação em Enfermagem e Saúde, Departamento de Saúde, Universidade Estadual do Sudoeste da Bahia (UESB) - Jequié (BA), Brasil.

${ }^{2}$ Núcleo de Estudos em Saúde da População, Departamento de Saúde, Universidade Estadual do Sudoeste da Bahia (UESB) - Jequié (BA), Brasil.

Trabalho realizado nas Estratégias de Saúde da Família no município de Ibicuí, localizado na Região Sudoeste da Bahia, Brasil.

Endereço para correspondência: Saulo Vasconcelos Rocha - Av. José Moreira Sobrinho, s/n, Jequiezinho - CEP: 45206-510 - Jequié (BA), Brasil -

Email: svrocha@uesb.edu.br

Fonte de financiamento: À Coordenação de Aperfeiçoamento de Pessoal de Nível Superior (CAPES), entidade financiadora da Bolsa de Mestrado.

Conflito de interesses: nada que declarar.
\end{abstract}




\section{INTRODUÇÃO}

O aumento da expectativa de vida, decorrente de mudanças demográficas e epidemiológicas, impactou no perfil de morbimortalidade da população ${ }^{1}$. No Brasil, as estimativas mostram que houve um crescimento acentuado da população idosa nas últimas décadas, passando de 9 para $12 \%$ da população geral $^{2}$.

Entre a população idosa, é muito comum a coexistência de múltiplas doenças crônicas, fato esse que a leva ao uso recorrente dos serviços de saúde e, consequentemente, à elevação dos gastos em saúde si,3, $^{1,4}$

A multimorbidade é um importante preditor de complicações e desfechos desfavoráveis em idosos 5 . Quando detectada precocemente, a presença de multimorbidade pode ter seus efeitos reduzidos por meio do tratamento adequado, garantindo, assim, maior sobrevida ${ }^{6}$.

Dos instrumentos utilizados na avaliação e no monitoramento do estado de saúde, o teste de Força de Preensão Manual (FPM) estima a força física global, além de ser utilizado para detectar outros desfechos em saúde, principalmente em idosos ${ }^{6-8}$.

Estudos recentes apontam que existe uma associação entre a FPM e a presença de doenças crônicas, indicando que a FPM é uma medida importante para ser considerada na avaliação e no monitoramento das doenças crônicas e das multimorbidades na população idosa ${ }^{8-10}$.

Na literatura brasileira, ainda são incipientes estudos que mostram a associação entre FPM, presença de doenças crônicas e multimorbidades. No entanto, os estudos encontrados indicam que a FPM tem associação com multimorbidades em idosos ${ }^{9,10}$.

Partindo desse pressuposto, o presente estudo objetivou selecionar, por meio da sensibilidade e da especificidade, os melhores pontos de corte para a força de preensão manual como discriminador de risco para presença de multimorbidade em idosos.

\section{MÉTODO}

Trata-se de um estudo de corte transversal. As informações utilizadas para este estudo foram extraídas do inquérito domiciliar intitulado "Monitoramento das Condições de Saúde de Idosos de um Município de Pequeno Porte: MONIDI", desenvolvido no mês de fevereiro de 2014.

O estudo foi realizado no município de Ibicuí, localizado na região sudoeste da Bahia. Sua população estimada para o ano de 2014 era de 16.640 habitantes, dos quais 2.124 eram idosos $^{11}$.

\section{Amostra}

A população-alvo do presente estudo foi constituída por 1.153 indivíduos com idade igual ou maior que 60 anos, cadastrados pela Estratégia Saúde da Família (ESF) do município de Ibicuí. No ano de 2014, 68,1\% da população residente tinha cobertura pela ESF. Para a determinação do tamanho da amostra, foram utilizados os critérios propostos por Luiz e Magnanini ${ }^{12}$ para populações finitas.

Os critérios de inclusão utilizados no estudo foram: indivíduos com idade igual ou maior que 60 anos, residentes nas zonas urbana e rural, cadastrados na ESF do município. Foram excluídos todos os indivíduos com diagnóstico de demência ou qualquer outro tipo de alteração cognitiva que comprometesse a veracidade das informações fornecidas.

Após os critérios de exclusão, foi realizado um sorteio aleatório dos usuários cadastrados nas Unidades de Saúde da Família (USF), considerando a distribuição proporcional pelo número de idosos cadastrados por USF e sexo dos usuários. A amostra foi obtida assumindo-se uma prevalência de 50\%, erro amostral de 3 p.p e nível de confiança de 95\% (Figura 1).

\section{Instrumento de pesquisa}

Foi utilizado instrumento de coleta denominado Instrumento de Avaliação da Saúde de Idosos (IASI), padronizado e validado previamente $^{13}$, que foi respondido pelos participantes na Unidade de Saúde em que os usuários estavam cadastrados ou no domicílio.

Assim, anteriormente à realização da coleta de dados, fez-se um estudo-piloto com uma população com características semelhantes aos indivíduos incluídos no presente estudo. Procedimentos padronizados foram adotados para garantir a qualidades das informações fornecidas tanto pelo questionário quanto pelas medidas antropométricas e de força muscular. A equipe de coleta recebeu treinamento prévio antes do início do estudo. Além disso, a coleta foi monitorada pelos coordenadores da pesquisa durante o período do estudo, e uma amostra de informações foi analisada regularmente com o propósito de identificar possíveis equívocos na coleta dos dados.

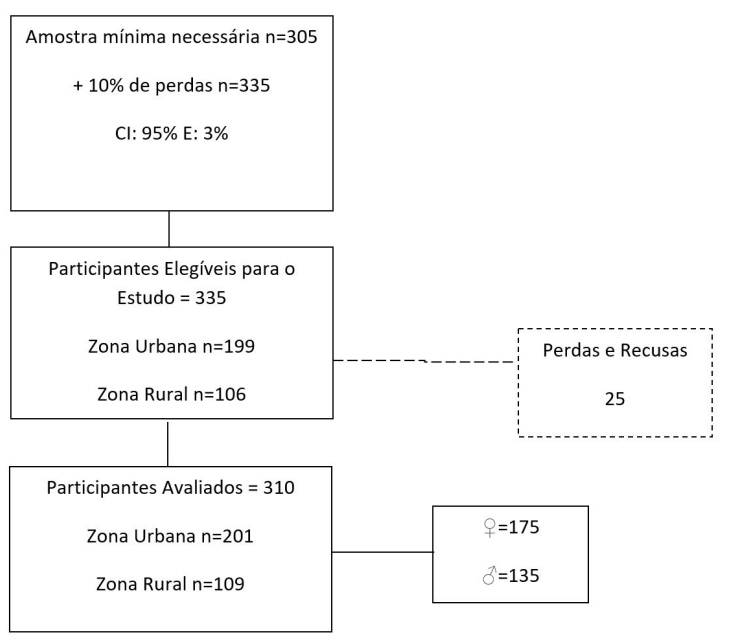

Figura 1. Esquema do processo de seleção e amostra do estudo em Ibicuí, Brasil, 2014 
As dimensões do instrumento de coleta utilizado no presente estudo foram compostas por blocos de informações, incluindo:

- Características sociodemográficas e informações pessoais: sexo, idade (em anos completos), situação conjugal (com companheiro e sem companheiro), nível de escolaridade (alfabetizado e não alfabetizado) e renda média mensal (em reais, relativa à renda média do idoso nos últimos 12 meses anteriores à coleta).

- Presença de multimorbidade: considerou-se como multimorbidade a presença de duas ou mais morbidades crônicas no mesmo indivíduo ${ }^{14}$. De acordo com o instrumento de coleta, a presença de doenças crônicas autorreferidas pelos entrevistados foi categorizada como "sim" e "não", e, assim, quantificado o número de morbidades por idoso, conforme doenças listadas a seguir:

- Doenças crônicas autorreferidas: diabetes, colesterol elevado, hipertensão, cardiopatia, embolia pulmonar, acidente vascular encefálico, câncer, artrite/artrose e Parkinson em conformidade com a Classificação Internacional de Doenças - CID-10 $0^{15}$;

- Força de Preensão Manual: avaliada por meio de um dinamômetro hidráulico Electronic Hand (E.Clear - Model: EH101), de acordo com o método padronizado por Vianna et al. ${ }^{16}$. $\mathrm{O}$ teste foi realizado com o indivíduo em pé, segurando o aparelho na posição ortostática, com braço de maior força em extensão, ao lado do corpo. Os participantes foram estimulados a desenvolver uma força máxima, após duas tentativas, com intervalo de 1 minuto entre elas. A média das duas tentativas (kgf) foi considerada para o estudo.

\section{Procedimentos de análise}

Para a análise estatística dos dados, foi utilizado o pacote estatístico Statistical Package for Social Sciences for Windows, versão $21.0^{17}$, com o qual se realizou a análise descritiva para todas as variáveis, sendo adotados, para análise e interpretação dos dados, o intervalo de confiança de $95 \%$ e o nível de significância de $5 \%$.

A análise das curvas Receiver Operating Characteristic (ROC) foi conduzida pelo Software MedCalc for Windows, versão $12.5^{18}$.

Os pontos de corte para FPM foram determinados por meio da análise das curvas ROC. Inicialmente, foi identificada a área total sob a curva ROC entre a FPM e a presença de multimorbidade, utilizando-se intervalo de confiança (IC) de 95\%. Quanto maior a área sob a curva ROC, maior o poder discriminatório da FPM para presença de multimorbidade. O IC determina se a capacidade preditiva da FPM não é em razão do acaso, e o seu limite inferior não deve ser menor do que 0,50 . Posteriormente, foram identificados os pontos de corte com seus respectivos valores preditivos, sensibilidades e especificidades.
Esta pesquisa seguiu os princípios éticos presentes na Declaração de Helsinque ${ }^{19}$ e na Resolução no 466/2012 do Conselho Nacional de Saúde ${ }^{20}$. Os protocolos de pesquisa foram avaliados e aprovados pelo Comitê de Ética em Pesquisa com Seres Humanos da Universidade Estadual do Sudoeste da Bahia (CAAE: 22969013.0.0000.0055). Todos os participantes assinaram o Termo de Consentimento Livre e Esclarecido (TCLE) antes de serem entrevistados.

\section{RESULTADOS}

A amostra do presente estudo foi composta por 310 idosos, com uma taxa de resposta de $92,5 \%$ e de perdas e recusas de $7,5 \%$.

No tocante às características sociodemográficas e às informações pessoais entre os entrevistados, houve predominância do sexo feminino (56,5\%); a média de idade foi de 71,62 $\pm 8,15$ anos, com destaque para a faixa etária compreendida entre 60-79 anos (83,9\%). A maioria dos indivíduos relatou ser alfabetizada $(56,1 \%)$, viver sem companheiro (51\%), com renda mensal média de $\mathrm{R} \$ 708,26 \pm 303,69$.

A prevalência geral de multimorbidade na população estudada foi de $70,6 \%$. A parcela mais expressiva de idosos relatou possuir quatro morbidades ou mais de maneira concomitante $(42,9 \%)$. As mulheres (49\%) apresentaram maior prevalência de multimorbidades quando comparadas aos homens $(31,3 \%)$. Identificou-se maior prevalência de multimorbidade entre os idosos com idade maior ou igual a 80 anos (40\%), não alfabetizados $(33,8 \%)$ e sem companheiro $(24,7 \%)$, com associação estatisticamente significante (Tabela 1).

Com relação às doenças autorreferidas, as mais prevalentes foram: hipertensão arterial sistêmica $(64,2 \%)$, dores na coluna $(64,2 \%)$, doenças cardiocirculatórias $(38,3 \%)$, varizes $(37,1 \%)$ e problemas respiratórios (35,5\%) (Tabela 1$)$.

A média geral da FPM na população do estudo foi de $21,22 \mathrm{kgf} \pm 8,4,(17,0 \mathrm{kgf} \pm 6,1$ e $26,6 \mathrm{kgf} \pm 8,0$ para os sexos feminino e masculino, respectivamente).

A Figura 2 demonstra a área sob a curva ROC entre a FPM e a presença de multimorbidade em idosos do sexo feminino. A área sob a curva ROC, para o sexo feminino, foi de 0,59 (IC $95 \%=0,52-0,67$ ). O ponto de corte de $15,35 \mathrm{kgf}$ apresenta o mais adequado equilíbrio entre sensibilidade $(69,7 \%)$ e especificidade $(52,2 \%)$.

$\mathrm{Na}$ Figura 3, observou-se a área sob a curva ROC entre a FPM e a presença de multimorbidade em idosos do sexo masculino. A área sob a curva ROC para o sexo masculino foi de 0,58 (IC $95 \%=0,50-0,67$ ). O ponto de corte de $24,8 \mathrm{kgf}$ apresenta o equilíbrio mais adequado entre sensibilidade (49,5\%) e especificidade $(68,4 \%)$.

As descrições das interações entre a FPM e a presença de multimorbidade foram expressas nas Figuras 4 e 5 para os sexos feminino e masculino, respectivamente. 
Tabela 1. Características da amostra do estudo, em Ibicuí, Brasil, 2014

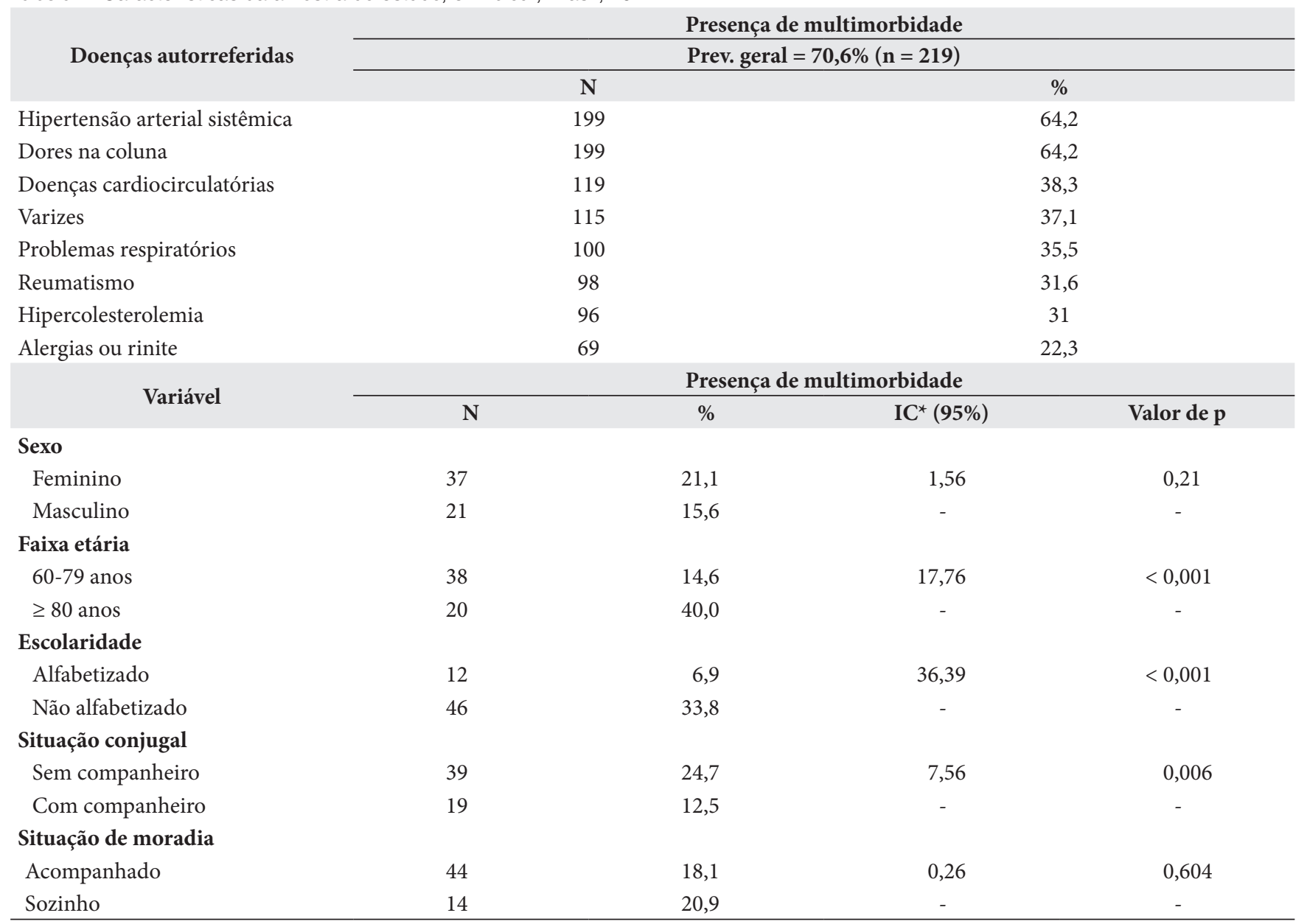

${ }^{\star}$ IC - intervalo de confiança

FPM

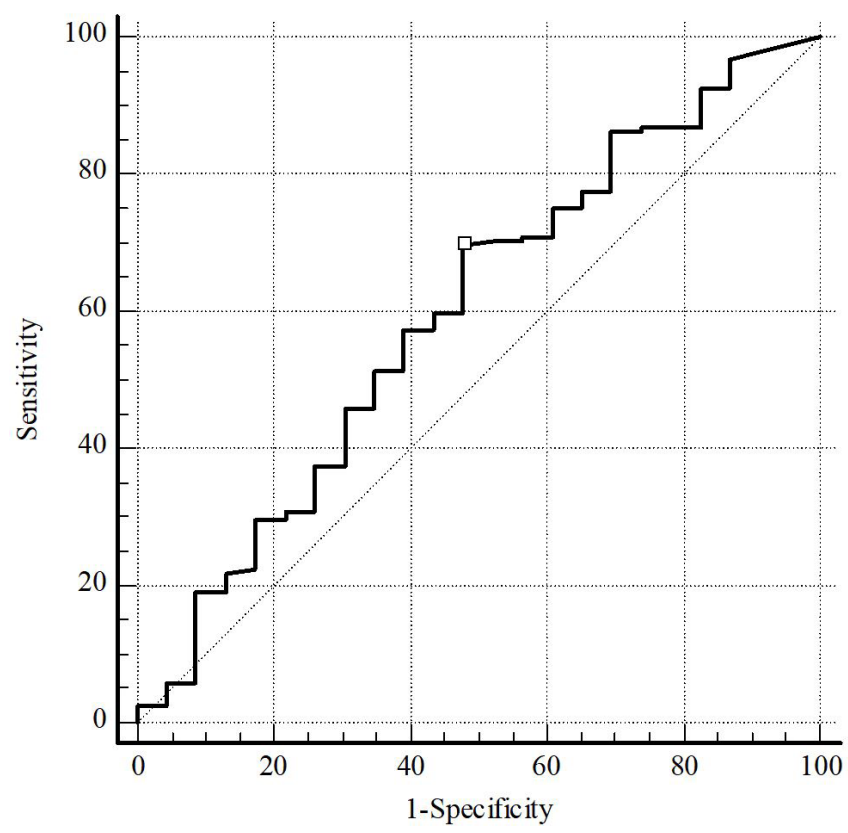

Figura 2. Área sob a curva ROC e IC $95 \%$ entre Força de Preensão Manual (FPM) e multimorbidade em indivíduos do sexo feminino, em Ibicuí, Brasil, 2014

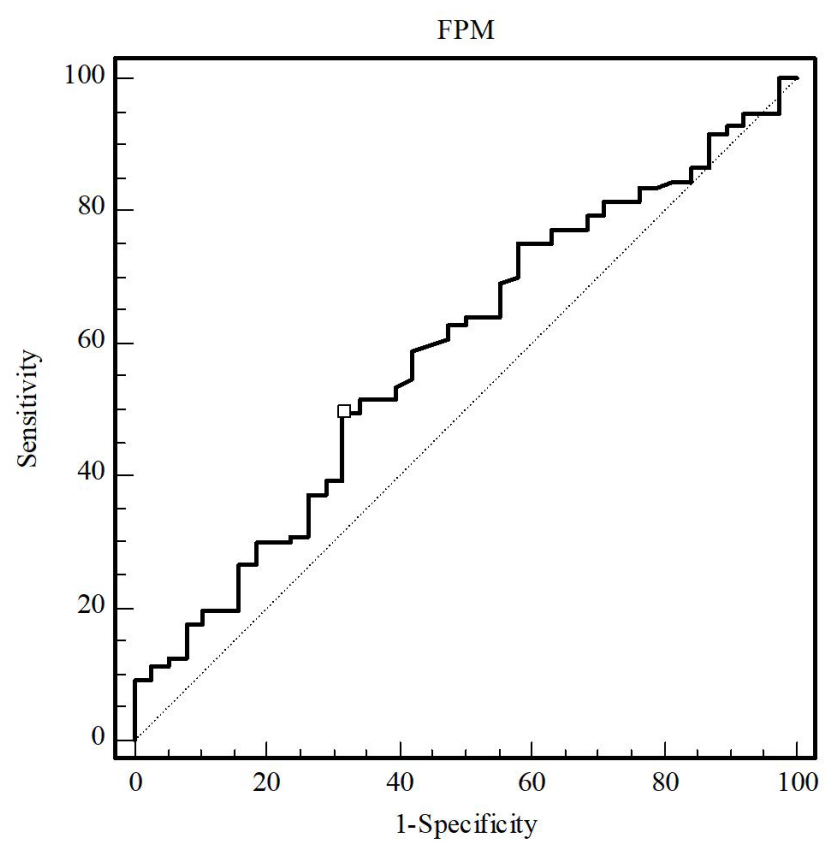

Figura 3. Área sob a curva ROC e IC 95\% entre Força de Preensão Manual (FPM) e multimorbidade em indivíduos do sexo masculino, em Ibicuí, Brasil, 2014 
FPM

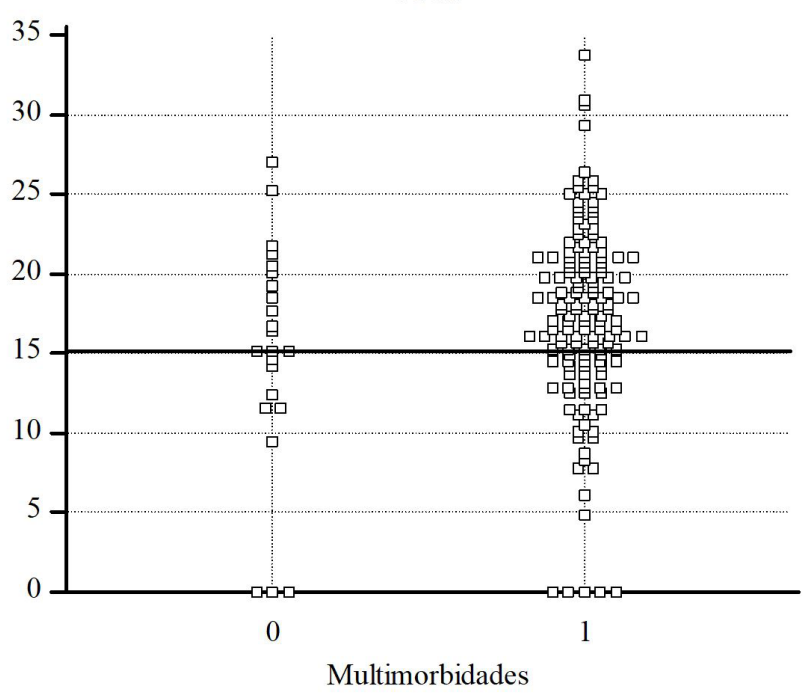

Figura 4. Gráfico de interação entre Força de Preensão Manual (FPM) e multimorbidade em indivíduos do sexo feminino, em Ibicuí, Brasil, 2014

FPM

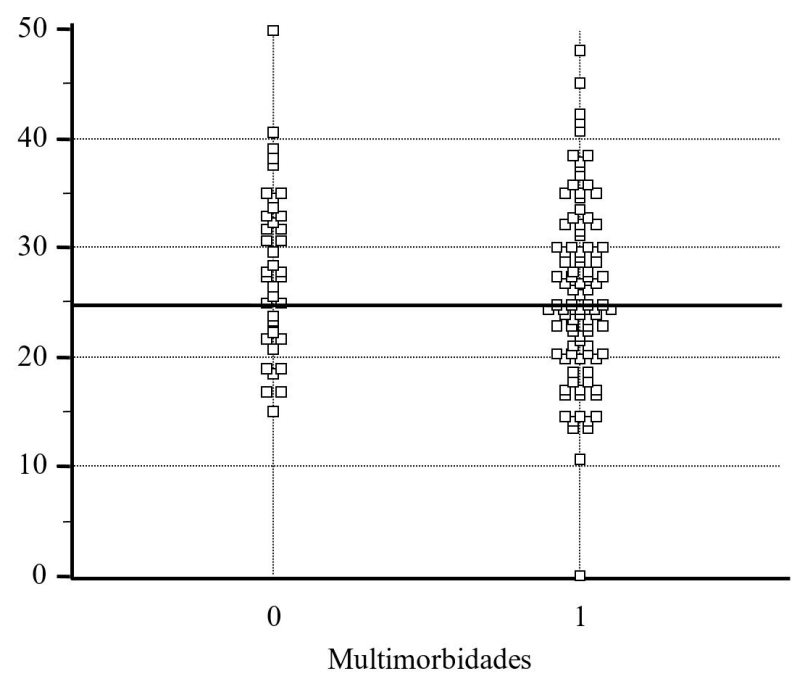

Figura 5. Gráfico de interação entre Força de Preensão Manual (FPM) e multimorbidade em indivíduos do sexo masculino, em Ibicuí, Brasil, 2014

\section{DISCUSSÃO}

Os resultados do presente estudo mostram que a FPM apresenta uma razoável capacidade discriminatória para a predição de multimorbidades em idosos, e os valores de 15,35 kgf para mulheres e 24,8 kgf para homens são os melhores pontos de corte encontrados, isto é, aqueles que demonstram o maior equilíbrio entre sensibilidade e especificidade.

A medida da FPM é um importante marcador de fragilidade, incapacidade, morbidade e mortalidade em idosos e tem sido cada vez mais utilizado em decorrência da sua capacidade de rastrear alterações relacionadas à idade no desempenho físico ${ }^{7,21}$.

Observou-se, no presente estudo, um desempenho satisfatório da FPM para discriminar a presença de multimorbidades, o que confirma o resultado de outros estudos que demonstraram que a FPM é um importante indicador da força muscular global, já que a redução da força nas extremidades superiores também representa fraqueza nos demais grupos musculares ${ }^{22}$.

Nesse sentido, achados da literatura mostraram que a redução dos escores de FPM estão relacionados à maior probabilidade de múltiplas doenças crônicas ${ }^{9,10}$. Entre as doenças crônicas relacionadas ao declínio da FPM, estão a hipertensão arterial, as dores musculares, a menor competência na execução das atividades cotidianas, os distúrbios de sono e a incapacidade física de modo geral2 ${ }^{23,24}$. Além disso, outras doenças também foram associadas aos baixos níveis de FPM, a exemplo de artrite, reumatismo e artrose em mulheres e de diabetes em homens?

A perda de força também interfere no equilíbrio dos idosos, favorecendo episódios de quedas, que é a segunda maior causa de morte nessa população, tornando um complicador à saúde deles e podendo levá-los à morte ou deixar sequelas, bem como prejuízos à capacidade funcional ${ }^{9,25}$.

Evidenciou-se, no presente estudo, que o valor médio da FPM foi significativamente maior entre o sexo masculino, quando comparado ao sexo feminino, confirmando os resultados encontrados na literatura ${ }^{8,10,26,27}$.

Observou-se também que grande parte dos idosos relatou possuir quatro ou mais morbidades concomitantemente, sendo as mais referidas a hipertensão e as dores na coluna. Resultados parecidos foram identificados em outros estudos ${ }^{28,29}$.

Levantamento realizado com idosos residentes em comunidade do Sul do Brasil identificou que a associação entre as condições crônicas e a redução da FPM ocorre de forma diferente entre homens e mulheres. No caso dos homens, a redução da FPM está associada à presença de diabetes, doença pulmonar crônica e doença arterial coronariana, com associação inversa entre número de doenças e redução da FPM. Já entre as mulheres, doenças, como câncer e depressão, estão associadas à redução da FPM sem relação significativa entre o número de doenças e agravos e a redução da $\mathrm{FPM}^{10}$.

Alterações hormonais, sobretudo pelos maiores níveis de testosterona e pela maior quantidade de massa muscular nos homens, e pela maior quantidade de gordura corporal nas mulheres ${ }^{30,31}$, podem explicar o maior desempenho de FPM nos homens.

Estudo realizado com idosos, residentes em Florianópolis, aponta níveis de força comparáveis aos detectados no presente estudo (17,9 kgf $\pm 5,4 \mathrm{em}$ mulheres e 29,3 kgf \pm 8,7 em homens). Verificou-se também redução da FPM média, em ambos os sexos, em idosos portadores de doenças crônicas. Em relação às multimorbidades, foi perceptível a redução da FPM média preditiva nos idosos de ambos os sexos enquadrados nessa categoria ${ }^{32}$. 
A literatura aponta que alterações hormonais, sobretudo pelos maiores níveis de testosterona e pela maior quantidade de massa muscular nos homens, podem explicar o maior desempenho de FPM observada no sexo masculino, enquanto, nas mulheres, isso se dá em decorrência da maior composição de gordura corporal ${ }^{30,31}$.

Com relação aos valores dos escores de FPM e à sua associação com doenças e agravos negativos à saúde, estudos sugerem que valores de FPM iguais ou inferiores a $20 \mathrm{kgf}$ (valor próximo ao observado no presente estudo como melhor ponto de corte para idosos do sexo masculino) estão relacionados à baixa condição de saúde e ao risco aumentado para dependência ${ }^{33}$. Ainda foi possível observar entre as mulheres do presente estudo valores de FPM inferiores ao recomendado. O valor de força encontrado pode ser levado em consideração para avaliação global da saúde das idosas pesquisadas.

Ao fazer a análise dos valores preditivos obtidos por meio da análise da sensibilidade, especificidade e prevalência, observou-se a consistência na análise da curva ROC, pois a elevada prevalência de multimorbidade na população estudada obteve maior valor preditivo positivo e menor valor preditivo negativo para ambos os sexos, demonstrando que, quanto mais frequente a morbidade na população estudada, maior é a probabilidade de encontrar verdadeiros positivos ${ }^{34}$.

Este estudo apresenta algumas limitações que devem ser consideradas. A amostra tomou como base apenas os idosos cadastrados na ESF. Apesar de a cobertura da ESF ser de 68,1\% no município, a amostra não é representativa dos idosos residentes em Ibicuí. Além disso, a avaliação da presença de doenças foi autorreferida e está sujeita a viés de memória.

Em contrapartida, a pesquisa possui relevância para a saúde pública, pois, além de seus dados terem sido obtidos por meio de questionário e testes padronizados, incluiu a população idosa de uma região pouco investigada no país.

Os resultados obtidos neste estudo apontam que o teste de preensão apresentou um desempenho satisfatório na discriminação da presença de multimorbidades nos idosos investigados.

Além disso, o estudo revelou dados significativos que interferem diretamente na saúde dos investigados, podendo nortear o direcionamento das ações locais em saúde, visando a um envelhecimento saudável.

Dessa forma, sugere-se a realização estudos com análise múltipla, a fim identificar os fatores associadas às morbidades encontradas na presente população e a confirmação de tais achados.

\section{REFERÊNCIAS}

1. Carneiro LAF, Campino ACC, Leite F, Rodrigues CG, Santos GMM, Silva ARA. Envelhecimento populacional e os desafios para o sistema de saúde brasileiro [Internet]. São Paulo: IESS; 2013 [citado em 2015 Ago 14]. Disponível em: www.iess.org.br/envelhecimentopop2013.pdf

2. Instituto Brasileiro de Geografia e Estatística. Projeção da população do Brasil e das Unidades da Federação [Internet]. Rio de Janeiro: IBGE; 2015 [citado em 2015 Out 21]. Disponível em: http://www.ibge.gov.br/apps/ populacao/projecao/

3. Silveira RE, Santos AS, Sousa MC, Monteiro TS Gastos relacionados a hospitalizações de idosos no Brasil. Einstein. 2013;11(4):514-20. http:// dx.doi.org/10.1590/S1679-45082013000400019.

4. Kane RL, Butler M. Are we ready for multimorbidity? Aging Health. 2012;8(6):635-6. http://dx.doi.org/10.2217/ahe.12.62.

5. Martins M, Blais R, Miranda NN. Evaluation of the Charlson comorbidity index among patients in Ribeirão Preto, São Paulo State, Brazil. Rev Saude Publica. 2008;24(3):643-52. http://dx.doi.org/10.1590/S0102311X2008000300018. PMid:18327452.

6. Sousa-Muñoz RL, Ronconi DE, Dantas GC, Lucena DMS, Silva IBA. Impacto de multimorbidade sobre a mortalidade em idosos: estudo de coorte pós-hospitalização. Rev Bras Geriatr Gerontol. 2013;16(3):579-89. http://dx.doi.org/10.1590/S1809-98232013000300015.

7. Lenardt MH, Grden CRB, Sousa JAV, Reche PM, Betiolli SE, Ribeiro DKMN. Fatores associados à diminuição de força de preensão manual em idosos longevos. Rev Esc Enferm USP. 2014;48(6):1006-12. http://dx.doi. org/10.1590/S0080-623420140000700007. PMid:25626499.
8. Yorke AM, Curtis AB, Shoemaker M, Vangsnes E. Grip strength values stratified by age, gender, and chronic disease status in adults aged 50 years and older. J Geriatr Phys Ther. 2015;38(3):115-21. http://dx.doi.org/10.1519/ JPT.0000000000000037. PMid:25594523.

9. Amaral CA, Portela MC, Muniz PT, Farias ES, Araújo TS, Souza OF Associação da força de preensão manual com morbidades referidas em adultos de Rio Branco, Acre, Brasil: estudo de base populacional. Cad Saúde Pública. 2015;31(6):1313-25. http://dx.doi.org/10.1590/0102-311X00062214.

10. Pessini J, Barbosa AR, Trindade EBSM. Doenças crônicas, multimorbidade e força de preensão manual em idosos de uma comunidade do Sul do Brasil. Rev Nutr. 2016;29(1):43-52. http://dx.doi.org/10.1590/167898652016000100005 .

11. Instituto Brasileiro de Geografia e Estatística. População de Ibicuí - Censo 2010. Rio de Janeiro: IBGE; 2010. [citado em 2014 Set 11]. Disponível em: http://www.ibge.gov.br/cidadesat

12. Luiz RR, Magnanini MF. A lógica da determinação do tamanho da amostra em investigações epidemiológicas. Cad de Saúde Coletiva. 2000;8(2):9-28.

13. Pedreira RBS, Rocha SV, Santos CA, Vanconcelos LRC, Reis MC. Content validity of the Geriatric Health Assessment Instrument. Einstein, 2016;14(2):158-77. http://dx.doi.org/10.1590/S1679-45082016AO3455.

14. World Health Organization. Chronic disease. 2014 [citado em 2014 Dez 7]. Disponível em: http://www.who.int/chp/en/

15. Organização Mundial da Saúde. Classificação Internacional de Doenças e problemas relacionados à saúde: décima revisão (CID-10). São Paulo: 
OMS; 1993. Centro Colaborador da OMS para Classificação de Doenças em Português.

16. Vianna LC, Oliveira BR, Araujo CG. Age-related decline in handgrip strength differs according to gender. J Strength Cond Res. 2007;21(4):13104. PMid: 18076278.

17. Windows. Statistical Package for Social Sciences - SPSS for Windows versão 21.0. USA; 2015.

18. MedCalc. MedCalc for Windows, version 12.5: statistical analyses were performed using. MedCalc Software. Ostend, Belgium: MedCalc; 2015 [citado em 2015 Ago 26]. Disponível em: https://www.medcalc.org/ download.php

19. Associação Médica Mundial. Declaração de Helsinque. Princípios éticos para as pesquisas médicas em seres humanos. Edimburgo, Escócia: Associação Médica Mundial; 2000 [citado em 2015 Ago 19]. Disponível em: http:// www.fcm.unicamp.br/fcm/sites/default/files/declaracao_de_helsinque.pdf

20. Brasil. Ministério da Saúde. Resolução nº 466, de 12 de dezembro de 2012. Diário Oficial da União. Brasília, 2012.

21. Confortin SC, Barbosa AR, Danielewicz AL, Meneghini V, Testa WL. Motor performance of elderly in a community in southern Brazil. Rev Bras Cineantropom Desempenho Hum. 2013;15(4):417-26.

22. Welmer AK, Kåreholt I, Angleman S, Rydwik E, Fratiglioni L. Can chronic multimorbidity explain the age-related differences in strength, speed and balance in older adults? Aging Clin Exp Res. 2012;24(5):480-9. PMid:22961066

23. Ribeiro LHM, Neri AL. Exercícios físicos, força muscular e atividades de vida diária em mulheres idosas. Cien Saude Colet. 2012;17(8):2169-80. http://dx.doi.org/10.1590/S1413-81232012000800027. PMid:22899157.

24. Virtuoso JF, Balbé GP, Hermes JM, Amorin EE Jr, Fortunato AR, Mazo GZ. Força de preensão manual e aptidões físicas: um estudo preditivo com idosos ativos. Rev Bras Geriatr Gerontol. 2014;17(4):775-84.

25. Carmo ÉA, Souza TS, Nery AA, Vilela ABA, Martins IE Jr. Trend of mortality from external causes in elderly. J Nurs UFPE on line. 2017;11(Suppl. 1):37482. http://dx.doi.org/10.5205/reuol.7995-69931-4-SM.1101sup201717.
26. Martin FG, Nebuloni CC, Najas MS. Correlação entre estado nutricional e força de preensão palmar. Rev Bras Geriatr Gerontol. 2012;15(3):493-504. http://dx.doi.org/10.1590/S1809-98232012000300010.

27. Silva NA, Menezes TN, Melo RLP, Pedraza DF. Força de preensão manual e flexibilidade e suas relações com variáveis antropométricas em idosos. Rev Assoc Med Bras. 2013;59(2):128-35. http://dx.doi.org/10.1016/j. ramb.2012.10.002. PMid:23582553.

28. Felipe LK, Zimmermann A. Doenças crônicas degenerativas em idosos: dados fisioterapêuticos. RBPS. 2011;24(3):221-7.

29. Bueno DR, Rosa CSC, Codogno JS, Freitas IF Jr, Monteiro HL. Nível de atividade física, comorbidades e idade. Motriz. 2013;19(3):S16-24. http:// dx.doi.org/10.1590/S1980-65742013000700004.

30. Ditroilo M, Forte R, Benelli P, Gambarara D, De Vito G. Effects of age and limb dominance on upper and lower limb muscle function in healthy males and females aged 40-80 years. J Sports Sci. 2010;28(6):667-77. http:// dx.doi.org/10.1080/02640411003642098. PMid:20397097.

31. Welmer AK, Kåreholt I, Angleman S, Rydwik E, Fratiglioni L. Can chronic multimorbidity explain the age-related differences in strength, speed and balance in older adults? Aging Clin Exp Res. 2012;24(5):480-9. PMid:22961066.

32. Confortin SC, Danielewicz AL, Antes DL, Ono LM, d'Orsi E, Barbosa AR. Associação entre doenças crônicas e força de preensão manual de idosos residentes em Florianópolis - SC, Brasil. Ciênc Saúde Coletiva. 2018;23(5):1675-85. http://dx.doi.org/10.1590/1413-81232018235.19742016.

33. Geraldes AAR, Oliveira ARM, Albuquerque RB, Carvalho JM, Farinatti PTV. A forca de preensão manual é boa preditora do desempenho funcional de idosos frágeis: um estudo correlacional múltiplo. Rev Bras Med Esporte. 2008;14(1):12-6. http://dx.doi.org/10.1590/S1517-86922008000100002.

34. Martinez EZ, Louzada-Neto F, Pereira BB. A curva ROC para testes diagnósticos. Cad Saude Colet. 2003;11(1):7-31.

Recebido em: Set. 30, 2017 Aprovado em: Maio 17, 2018 